\title{
openheart Benefit of statin pretreatment in prevention of contrast-induced nephropathy in different adult patient population: systematic review and meta-analysis
}

\author{
Nirmal Singh, ${ }^{1}$ Justin Z Lee, ${ }^{2}$ Jennifer J Huang, ${ }^{2}$ See Wei Low, ${ }^{1}$ Carol Howe, ${ }^{3}$ \\ Anil Pandit, ${ }^{4}$ Prakash Suryanarayana, ${ }^{1}$ Kwan S Lee ${ }^{1}$
}

To cite: Singh N, Lee JZ, Huang JJ, et al. Benefit of statin pretreatment in prevention of contrastinduced nephropathy in different adult patient population: systematic review and meta-analysis. Open Heart 2014;1:e000127. doi:10.1136/openhrt-2014000127

- Additional material is published online only. To view please visit the journal (http://dx.doi.org/10.1136/ openhrt-2014-000127)

Received 25 March 2014 Revised 3 June 2014 Accepted 15 July 2014
CrossMark

For numbered affiliations see end of article.

Correspondence to Dr Kwan S Lee; klee@shc.arizona.edu

\section{ABSTRACT}

Objective: Previous studies have suggested that statin pretreatment prevents contrast-induced nephropathy (CIN). However, single randomised trials are limited in their number of patients. This meta-analysis aims to assess the role of statin use in CIN prevention, as well as to determine patient subgroups that will benefit from statin pre-treatment.

Methodology: We searched PubMed, EMBASE, Web of science and the Cochrane Central Register of Controlled Trials databases for randomised controlled trials (RCT) comparing statin pretreatment versus placebo for preventing CIN. Our main outcome was the risk of CIN within 1-5 days after contrast administration.

Results: Data analysed from nine randomised studies with a total of 5143 patients, where 2559 received statins and 2584 received placebo, showed that statin pretreatment was associated with significant reduction in risk of $\mathrm{CIN}(\mathrm{MH}-\mathrm{RR}=0.47,95 \% \mathrm{Cl} 0.34$ to 0.64 , $Z=4.49, p<0.00001)$. This beneficial effect of statin was also seen in patients with baseline renal impairment (MH-RR=0.46, 95\% Cl 0.29 to $0.72, p=0.0008$ ) and also those who were cotreated with NAC (MH-RR $=0.46$, $95 \% \mathrm{Cl} 0.25$ to $0.83, \mathrm{p}=0.01$ ).

Conclusions: Statin pretreatment leads to significant reduction in CIN, and should be strongly considered in all patients who are planned for diagnostic and interventional procedures involving contrast-media administration.

\section{INTRODUCTION}

Contrast-induced nephropathy (CIN) or contrast-induced acute kidney injury (CI-AKI) is the third most common cause of acute renal injury in the hospital setting. ${ }^{2}$ CIN is defined as an increase of serum creatinine of $0.5 \mathrm{mg} / \mathrm{dL}$ or a $25 \%$ relative increase in creatinine at $48 \mathrm{~h}$ after contrast exposure. ${ }^{3}$ CIN has been associated with poor short-term and long-term outcomes. ${ }^{4}$ Patients who develop CIN are predisposed to a greater risk of

\section{KEY MESSAGES}

What is already known about this subject?

- Previous studies have suggested that statin pretreatment prevents contrast-induced nephropathy (CIN).

- However, single randomized trials are limited in their number of patients, and there is still a lack of conclusive evidence on statin pre-treatment to prevent CIN.

What does this study add?

- This meta-analysis of nine randomized trials demonstrated that statin pre-treatment leads to significant reduction in CIN in patients undergoing coronary angiography, and is independent of renal impairment, type of contrast used, and concomitant $\mathrm{N}$-acetyl cysteine therapy.

How might this impact on clinical practice?

- Our findings suggest strong consideration of statin pre-treatment in all patients who are planned for diagnostic and interventional procedures involving contrast-media administration.

- This meta-analysis sets a platform for future pharmacological guidelines and clinical trials, as well as highlights the need for trials studying statin's efficacy in other non-cardiac contrast procedures such as radiological imaging.

developing chronic kidney disease (CKD) in the long term. ${ }^{5}$ This condition is also associated with increased morbidity, lengthened hospital stays and increased healthcare costs. ${ }^{6}$ Therefore, increasing efforts have been made to identify effective preventive strategies for CIN. The best approach to prevent CIN remains unclear. Current recommendations only include adequate preparatory hydration. $^{7}$ There is still a lack of conclusive evidence in other strategies such as administration of $\mathrm{N}$-acetylcysteine (NAC) as 
an antioxidant, sodium bicarbonate-based hydration and consideration of the type of contrast used. ${ }^{3}$

The pathophysiology of CIN involves renal vasoconstriction, inflammation and oxidative stress. ${ }^{8}$ These mechanisms have led to the proposed use of hydroxymethylglutaryl coenzyme A reductase inhibitors, otherwise known as statins, for the prevention of CIN.

There have been multiple randomised controlled trials (RCTs) performed to evaluate the efficacy of statin pretreatment in the prevention of CIN, with earlier trials failing to strongly demonstrate the beneficial effects of statin. Multiple meta-analyses have examined the role of statin pretreatment, but they had limited, variable and conflicting conclusions given the limited number of RCTs performed. ${ }^{9-12}$ Recently, the results of two large RCTs were published, ${ }^{13} 14$ adding more data into the existing pool and possibly providing new insights into the efficacy of statin to prevent contrast-nephropathy.

We therefore conducted a meta-analysis of RCTs to further evaluate the efficacy of statin pretreatment for prevention of CIN, as well as to ascertain its effectiveness in various patient subgroups.

\section{METHODS}

\section{Search strategy}

A systematic literature review was planned and performed using methods specified in the Preferred Reporting Items for Systematic Reviews and Meta-Analyses (PRISMA) guidelines for systematic review. Both controlled vocabulary terms (eg, MeSH) and key words were used to search the following databases for articles related to the role of statins in preventing or ameliorating CIN: MEDLINE/PubMed, EMBASE, Cochrane Library, Web of Science and ClinicalTrials.gov. Literature searches were completed in March, 2014. The complete MEDLINE/PubMed Search strategy, on which the other database searches were also built, is available in online supplementary appendix A. Reference lists of citations to the ultimately included articles were also searched for articles that would meet inclusion criteria, as was the grey literature, including the websites of national and international nephrology societies.

\section{Study selection, data extraction and quality assessment}

Our prespecified inclusion criteria were as follows: (1) randomised controlled trials, (2) treatment arm containing any of the available statins alone or with NAC, (3) control arm containing placebo or NAC, (4) both arms administer NAC if the study incorporated NAC, (5) intravenous or intra-arterial iodinated contrast medium. Our primary outcome was the development of contrast-induced nephropathy, defined as an absolute increase of serum creatinine of $0.5 \mathrm{mg} / \mathrm{dL}$ or an increase in baseline serum creatinine level of $25 \%$ within $24 \mathrm{~h}$ to 5 days after exposure to contrast medium or serum Cystatin concentration $>10 \%$ within $24 \mathrm{~h}$ after exposure to contrast medium. ${ }^{15}$ Secondary outcome measurement was the need for dialysis within $24 \mathrm{~h}$ to 5 days after contrast medium exposure.

Two independent reviewers performed the study selection (NS, JL). In case of disagreements, a third reviewer $(\mathrm{CH})$ cast the deciding vote. Titles and abstracts of retrieved references were screened for inclusion and full texts of potential articles were further analysed to see if they met inclusion criteria. Case reports, letters, systematic reviews/meta-analyses and data published in the form of abstracts without peer-reviewed publication of the manuscripts were excluded. Articles not in English were not automatically excluded though four non-English articles were ultimately excluded due to the absence of adequate translation. Both individuals who collected the data used the following study specific characteristics, including study name, sample size of treatment group and control group per intention to treat analysis, event number and rate in both the treatment group and control group, statistical effect estimates used in the individual studies, type of statin used, statin protocol used, hydration protocol, NAC use, type and amount of contrast medium used, procedures which patients underwent, inclusion and exclusion criteria of individual studies, CIN definition prespecified in individual studies, rate of adverse events in treatment group and control group, and baseline patient characteristics each study including presence of diabetes, history of statin use and degree of renal impairment. Quality assessment was judged on allocation concealment, randomisation, blinding, completion of follow-up and intention-to-treat analysis. ${ }^{16}$ Study quality was assessed following the pattern of PRISMA guidelines and explanation. ${ }^{17}$ Attempts were made to contact the corresponding authors of included studies for any additional information, which was deemed relevant to this meta-analysis.

\section{Statistical analysis}

Statistical analyses were performed using Review Manager V.5.2.9 software (available from The Cochrane Collaboration). A study or trial level pooled analysis of the included RCT's was performed to evaluate the effect of pretreatment with statin on prevention of CIN. The analysis was performed according to the intention-to-treat strategy. Mantel Haenszel risk ratio (MH RR) with $95 \%$ CI was calculated. The $\chi^{2}$ statistic was calculated and a formal test of heterogeneity was conducted. The $\mathrm{I}^{2}$ index was used to summarise the proportion of the total variability in the estimates due to between-study variation. We regarded $\mathrm{I}^{2}$ of less than $25 \%, 25-50 \%$ and $>50 \%$ as low, moderate, and high amounts of heterogeneity, respectively. ${ }^{18}$ Random-effects model rather than the fixed-effects model was used to assess the overall estimate. ${ }^{19}$ We assessed for potential publication bias by using the funnel plots of SE of RR versus RR. ${ }^{20}$ Subgroup analysis was conducted based on renal impairment of participants, type of contrast medium (iodixanol (iso-osmolar contrast) or noniodixanol (low-osmolar contrast)), history of diabetes 
mellitus (with or without diabetes mellitus), type of statin used (atorvastatin or rosuvastatin), and the addition of NAC (with or without NAC). We also analysed the relative risk of acute renal failure requiring dialysis. All the tests were two tailed and a $p$ value less than 0.05 was regarded as significant in this meta-analysis.

\section{RESULTS}

\section{Baseline characteristics}

A total of 2173 potentially relevant citations were identified. Figure 1 shows our search strategy, which yielded nine randomised controlled trials with 5143 patients. ${ }^{131421-26}$ A total 2559 patients were randomised to receive statins, and 2584 patients to receive the placebo or NAC alone.

\section{Patient characteristics and interventions}

Tables 1-3 summarise the characteristics of the nine included RCTs. Table 4 displays the quality assessment of the included studies.

Among the nine RCTs, Jo et $a l^{26}$ used $40 \mathrm{mg}$ of simvastatin as a treatment arm against placebo while six other studies $^{21-25} 27$ used atorvastatin; $80 \mathrm{mg}$ atorvastatin was used in five studies $^{22-25}$ and $40 \mathrm{mg}$ was used in one study. $^{21}$ Two recent trials used rosuvastatin in different doses, with Han et $a l^{13}$ using $10 \mathrm{mg}$ and Leoncini et al $\mathrm{l}^{14}$ using $40 \mathrm{mg}$. Both studies showed significant beneficial effect of statin pretreatment. In terms of contrast medium, four studies 21242527 used non-ionic lowosmolar contrast medium and the rest of the studies $^{13} \quad 1423 \quad 26$ used non-ionic iso-osmolar contrast medium.

The contrast procedure performed were percutaneous coronary intervention (PCI) in two studies, ${ }^{24} 27$ coronary angiography or percutaneous coronary in three studies, ${ }^{14} 2223$ coronary angiography only in three studies, ${ }^{21} 2526$ and coronary angiography, percutaneous coronary intervention or peripheral arterial angiography in one study. ${ }^{13}$

The majority of the studies used serum creatinine increase of $>0.5 \mathrm{mg} / \mathrm{dL}$ within $24 \mathrm{~h}$ to 5 days as their definition of CIN with exception of two studies. ${ }^{21} 23$ Quintavalle et $a t^{23}$ used serum Cystatin C increase (10\%) within $24 \mathrm{~h}$ of contrast medium administration and Acikel et $a l^{21}$ used combination of serum creatinine, Cystatin $\mathrm{C}$ and glomerular filtration rate as the definition for CIN.

The hydration protocol with normal saline was standard in all the studies along with the use of NAC in four studies. $^{1422} 2325$

One similarity among all the studies was the inclusion of patients with diabetes in both arms of the RCTs. Of the nine studies, six studies ${ }^{13} 1422-2426$ had patients with chronic kidney disease with creatinine clearance $<60 \mathrm{~mL} / \mathrm{min}$ or glomerular filtration rate (GFR) $<60 \mathrm{~mL} / \mathrm{min} / 1.73 \mathrm{~m}^{2}$ and two studies ${ }^{21} 25$ excluded patients with GFR $<70 \mathrm{~mL} / \mathrm{min} / 1.73 \mathrm{~m}^{2}$. Li et $a l^{27}$ also excluded patients with history of renal dysfunction.

The primary end point of eight out of the nine studies was CIN, defined as an increase in serum creatinine by more than $25 \%$ or $0.5 \mathrm{mg} / \mathrm{dL}$ within 3 days, or serum Cystatin concentration $>10 \%$ within $24 \mathrm{~h}$ of the

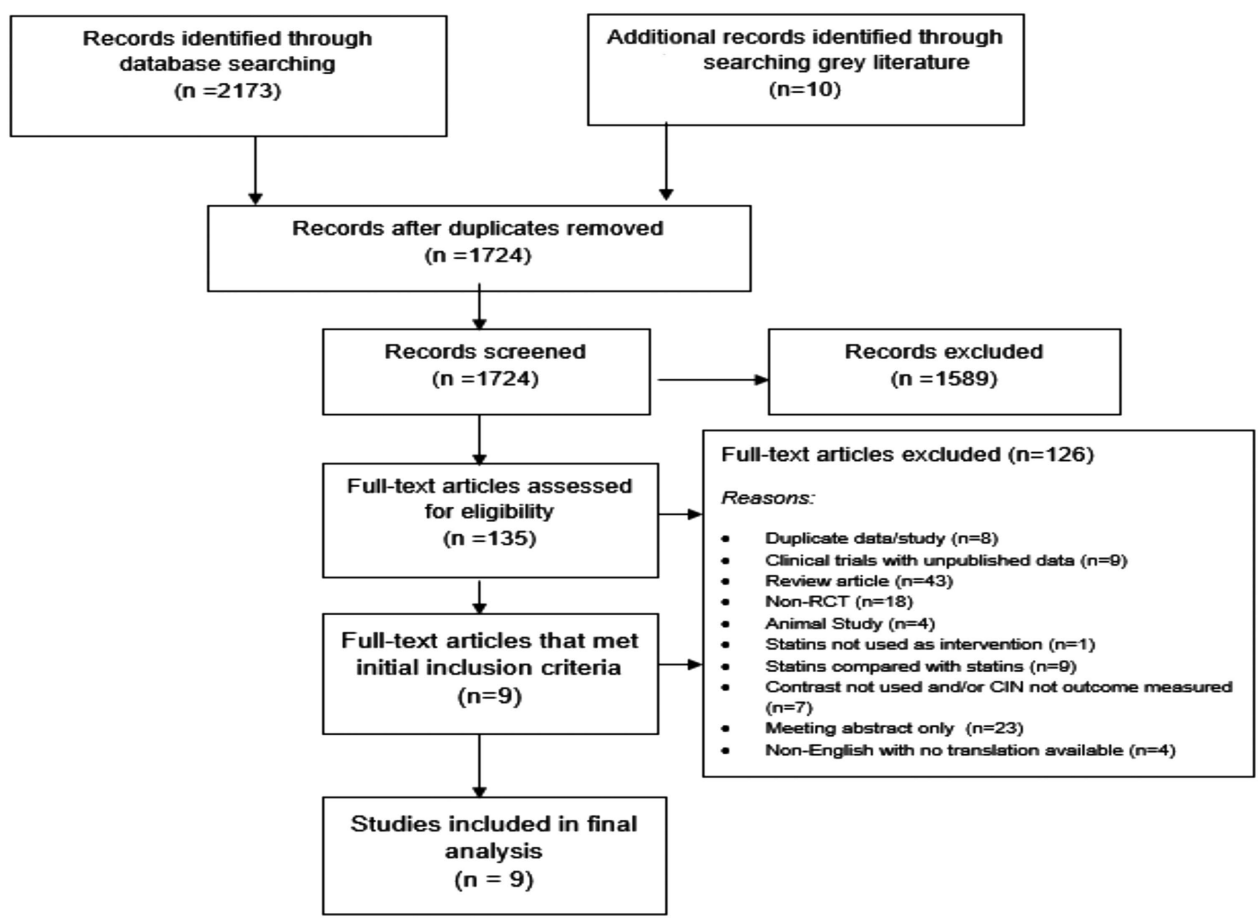

Figure 1 Study selection diagram. 
Table 1 General characteristics of included studies

\begin{tabular}{|c|c|c|c|c|c|c|c|}
\hline \multirow{2}{*}{$\begin{array}{l}\text { Study } \\
\text { (reference) }\end{array}$} & \multicolumn{2}{|c|}{$\begin{array}{l}\text { Sample } \\
\text { size (N) }\end{array}$} & \multirow[b]{2}{*}{ Definition of CIN } & \multirow{2}{*}{$\begin{array}{l}\text { Time of } \\
\text { definition }\end{array}$} & \multicolumn{2}{|c|}{ Events (n) } & \multirow{2}{*}{$\begin{array}{l}\text { Renal function defining } \\
\text { inclusion or exclusion }\end{array}$} \\
\hline & Statin & Control & & & Statin & Control & \\
\hline Acikel et $a P^{21}$ & 80 & 80 & $\begin{array}{l}\text { Increase in S Cr or serum } \\
\text { Cystatin C or GFR }\end{array}$ & Within $48 \mathrm{~h}$ & 0 & 1 & $\begin{array}{l}\text { Exclusion: CRF requiring } \\
\text { dialysis } \\
\text { and/or mod-severe decrease in } \\
\text { GFR }<60 \mathrm{~mL} / \mathrm{min} / 1.73 \mathrm{~m}^{2}\end{array}$ \\
\hline Han et $a l^{13}$ & 1498 & 1500 & $\begin{array}{l}\mathrm{S} \mathrm{Cr} \geq 0.5 \mathrm{mg} / \mathrm{dL} \text { or } 25 \% \\
\text { above baseline }\end{array}$ & Within $72 \mathrm{~h}$ & 34 & 58 & $\begin{array}{l}\text { Inclusion: Stage } 2 \text { or stage } 3 \\
\text { CKD } \\
\text { Exclusion: Stage } 0,1,4 \text { or } 5 \\
\text { CKD }\end{array}$ \\
\hline Jo et $a^{\text {R6 }}$ & 118 & 118 & $\begin{array}{l}\text { Increase of either } \geq 25 \% \\
\text { or } 0.5 \mathrm{mg} / \mathrm{dL} \text { in } \mathrm{S} \mathrm{Cr}\end{array}$ & Within $48 \mathrm{~h}$ & 3 & 4 & $\begin{array}{l}\text { Inclusion: } \mathrm{Cr} . \mathrm{Cl}<60 \mathrm{~mL} / \mathrm{min} \text { or } \\
\text { baseline } \mathrm{S} \mathrm{Cr} \geq 1.1 \mathrm{mg} / \mathrm{dL}\end{array}$ \\
\hline Leoncini et $a l^{14}$ & 252 & 252 & $\begin{array}{l}\mathrm{S} \mathrm{Cr} \geq 0.5 \mathrm{mg} / \mathrm{dL} \text { or } \\
\geq 25 \% \text { above baseline }\end{array}$ & Within $72 \mathrm{~h}$ & 17 & 38 & $\begin{array}{l}\text { Exclusion: } A R F \text { or ESRD } \\
\text { requiring dialysis or } \mathrm{S} \mathrm{Cr} \\
\geq 3 \mathrm{mg} / \mathrm{dL}\end{array}$ \\
\hline Li et $a f^{77}$ & 78 & 83 & $\begin{array}{l}\mathrm{S} \mathrm{Cr}>0.5 \mathrm{mg} / \mathrm{dL} \text { or } 25 \% \\
\text { above baseline }\end{array}$ & Within $24 \mathrm{~h}$ & 2 & 13 & $\begin{array}{l}\text { Exclusion: } h / o \text { renal } \\
\text { dysfunction, patient on dialysis } \\
\text { treatment }\end{array}$ \\
\hline Ozhan et $a R^{25}$ & 60 & 70 & $\begin{array}{l}\text { Increase in } \mathrm{S} \mathrm{Cr}>0.5 \\
\mathrm{mg} / \mathrm{dL} \text { or }>25 \% \text { from } \\
\text { baseline }\end{array}$ & Within $48 \mathrm{~h}$ & 2 & 7 & $\begin{array}{l}\text { Exclusion: (e)GFR }<70 \mathrm{~mL} / \mathrm{min} \\
\text { or pre-procedural } \\
\mathrm{S} \mathrm{Cr}>1.5 \mathrm{mg} / \mathrm{dL}\end{array}$ \\
\hline Patti et $a R^{4}$ & 120 & 121 & $\begin{array}{l}\text { Increase in } \mathrm{S} \mathrm{Cr} \\
\geq 0.5 \mathrm{mg} / \mathrm{dL} \text { or } 25 \% \text { from } \\
\text { baseline }\end{array}$ & Within $48 \mathrm{~h}$ & 6 & 16 & $\begin{array}{l}\text { Exclusion: Renal failure with } \\
\mathrm{S} \mathrm{Cr}>3 \mathrm{mg} / \mathrm{dL}\end{array}$ \\
\hline $\begin{array}{l}\text { Quintavalle } \\
\text { et } a^{23}\end{array}$ & 202 & 208 & $\begin{array}{l}\text { Increase }>10 \% \text { of } S \\
\text { Cystatin C level }\end{array}$ & Within $24 \mathrm{~h}$ & 9 & 37 & $\begin{array}{l}\text { Inclusion: Estimated GFR } \\
<60 \mathrm{~mL} / \mathrm{min} / 1.73 \mathrm{~m}^{2} . \text { Patients } \\
\text { with dialysis were excluded }\end{array}$ \\
\hline Toso et $a^{R^{2}}$ & 151 & 152 & $\begin{array}{l}\text { Increase of S Cr } \\
\geq 0.5 \mathrm{mg} / \mathrm{dL}\end{array}$ & $\begin{array}{l}\text { Within } \\
5 \text { days }\end{array}$ & 15 & 16 & $\begin{array}{l}\text { Inclusion: Baseline } \mathrm{Cr} \mathrm{Cl} \\
<60 \mathrm{~mL} / \mathrm{min} \text { (CGF) }\end{array}$ \\
\hline
\end{tabular}

administration of contrast medium in the absence of alternative explanations. ${ }^{28}$ In the Jo $e t a l^{26}$ study, change in serum creatinine level was the primary end point and incidence of CIN was the secondary end point.

\section{Outcomes}

Based on pooled estimates across the nine randomised controlled trials, there was a significant benefit associated with statin treatment $(\mathrm{MH}-\mathrm{RR}=0.47,95 \%$ CI 0.34 to $0.64, \mathrm{p}<0.00001$ ). Thus, statin use, irrespective of type of statin, has a beneficial role in preventing CIN. We drew our conclusions using the random-effects model for this analysis given the clinical and methodological heterogeneity of the RCTs included in the meta-analysis. ${ }^{19}$

The Forest plot in figure 2 shows the results of studies pooled in the meta-analysis.

There was moderate heterogeneity among the studies using random method $\left(\mathrm{I}^{2}=29 \%, \chi^{2}=11.24, \mathrm{df}=8, \mathrm{p}=0.19\right)$. The $\mathrm{I}^{2}$ measure was used to quantify heterogeneity as it is independent of the number of studies in the meta-analysis and has better power as compared to using the $\chi^{2}$ method. ${ }^{29} 30$
Sensitivity analysis measured the effect of individual studies on the summary effect size. It showed that the summary effect was statistically significant $(\mathrm{MH}-\mathrm{RR}=0.40$, $95 \%$ CI 0.28 to $0.59, \mathrm{p}<0.00001$, benefit by $7 \%$ ). After removing studies by Ozhan et $a l^{25}$ Toso $e t a l,{ }^{22} \mathrm{Jo} e t a l^{26}$ study and Acikel et $a l^{21}$ the statistical significance improved. Publication bias was assessed by Funnel plot (figure 3) with $\mathrm{SE}$ of $\log \mathrm{RR}$ against RR. It shows the symmetrical distribution of the plot around the summary effect size showing no publication bias.

\section{Subgroup analysis}

There is clinical and methodological diversity in the studies included in this meta-analysis. This issue was addressed by using subgroup analysis as prespecified in the methods section.

Renal impairment

Out of nine clinical trials, six studies ${ }^{13} \quad 14 \quad 22-24 \quad 26$ included patients with pre-existing renal impairment. Among them, two ${ }^{14}{ }^{24}$ have patients with normal renal function and the other five $\begin{array}{llll}13 & 22 & 26 & 26 \\ (8,9,12,16)\end{array}$ included only patients without renal impairment at the baseline. Ozhan et $a l^{21}{ }^{25}$ excluded patients with GFR 
Table 2 Protocol characteristics of included studies

\begin{tabular}{|c|c|c|c|c|c|c|c|c|}
\hline \multirow{2}{*}{$\begin{array}{l}\text { Study } \\
\text { (reference) }\end{array}$} & \multirow[b]{2}{*}{ Statin type } & \multirow[b]{2}{*}{ Statin protocol } & \multirow[b]{2}{*}{ Control } & \multirow{2}{*}{$\begin{array}{l}\text { Contrast } \\
\text { agent }\end{array}$} & \multicolumn{2}{|c|}{$\begin{array}{l}\text { Median contrast } \\
\text { volume, } \mathrm{mL}\end{array}$} & \multirow[b]{2}{*}{ Procedure } & \multirow[b]{2}{*}{ Hydration procedure } \\
\hline & & & & & Statin & Control & & \\
\hline $\begin{array}{l}\text { Acikel } \\
\text { et } a^{P^{1}}\end{array}$ & Atorvastatin & $\begin{array}{l}40 \mathrm{mg} / \text { day atorvastatin } \\
\text { started } 3 \text { days before CAG } \\
\text { and continued for } 48 \mathrm{~h} \text { after } \\
\text { procedure }\end{array}$ & Placebo & lohexol & 105 & 103 & CAG & $\begin{array}{l}\text { IV } 0.9 \% \mathrm{NaCl} \text { at } 1 \mathrm{~mL} / \mathrm{kg} / \mathrm{h} \\
\text { starting } 4 \mathrm{~h} \text { before and } \\
\text { continuing until } 24 \mathrm{~h} \text { after } \\
\mathrm{CM} \text { exposure }\end{array}$ \\
\hline Han et $a l^{13}$ & Rosuvastatin & $\begin{array}{l}10 \mathrm{mg} / \text { day rosuvastatin for } \\
5 \text { days ( } 2 \text { days before and } \\
3 \text { days after procedure) }\end{array}$ & Placebo & Iodixanol & 120 & 110 & $\begin{array}{l}\text { Coronary/peripheral } \\
\text { arterial angiography } \\
\text { with or without } \\
\text { percutaneous } \\
\text { intervention }\end{array}$ & $\begin{array}{l}\text { IV } 0.9 \% \mathrm{NaCl} \text { at } 1 \mathrm{~mL} / \mathrm{kg} / \mathrm{h} \\
\text { started } 12 \mathrm{~h} \text { before and } \\
\text { continued for } 24 \mathrm{~h} \mathrm{CM} \\
\text { administration }\end{array}$ \\
\hline Jo et $a^{26}$ & Simvastatin & $\begin{array}{l}160 \mathrm{mg} \text { total, } 40 \mathrm{mg} \text { orally } \mathrm{q} \\
12 \mathrm{~h} \text { starting evening before } \\
\text { and ending morning after } \\
\text { the procedure }\end{array}$ & Placebo & lodixanol & $173 \pm 99.3$ & $190.9 \pm 133.5$ & CAG & $\begin{array}{l}\text { IV } 0.45 \% \mathrm{NaCl} \text { at } 1 \mathrm{mg} / \mathrm{kg} / \mathrm{h} \\
\text { for } 12 \mathrm{~h} \text { before and } 12 \mathrm{~h} \\
\text { after the procedure }\end{array}$ \\
\hline $\begin{array}{l}\text { Leoncini } \\
\text { et } \mathrm{al}^{14}\end{array}$ & Rosuvastatin & $\begin{array}{l}40 \mathrm{mg} \text { rosuvastatin on } \\
\text { admission, followed by } \\
20 \mathrm{mg} / \text { day plus } 1200 \mathrm{mg} \\
\mathrm{NAC} \text { twice daily from the } \\
\text { day before through the day } \\
\text { after angiography }\end{array}$ & $\begin{array}{l}\text { Placebo plus } 1200 \mathrm{mg} \\
\text { NAC twice daily from } \\
\text { the day before } \\
\text { through the day after } \\
\text { angiography }\end{array}$ & Iodixanol & $183 \pm 80$ & $172 \pm 72$ & $\mathrm{CAG} \pm \mathrm{PCl}$ & $\begin{array}{l}\text { IV } 0.9 \% \mathrm{NaCl} \text { at } 1 \mathrm{~mL} / \mathrm{kg} / \mathrm{h} \\
\text { for } 12 \mathrm{~h} \text { before and after } \\
\text { procedure }\end{array}$ \\
\hline Li et $a P^{7}$ & Atorvastatin & $\begin{array}{l}\text { High-dose atorvastatin } \\
80 \mathrm{mg} \text { prior to procedure } \\
\text { and } 40 \mathrm{mg} \text { every day } \\
\text { thereafter }\end{array}$ & Placebo & Iopromide & $100 \pm 25.9$ & $103.6 \pm 26.2$ & $\mathrm{CAG}$ and $\mathrm{PCl}$ & $\begin{array}{l}\text { IV } 0.9 \% \mathrm{NaCl} \text { at } 1 \mathrm{~mL} / \mathrm{kg} / \mathrm{h} \\
\text { before the procedure and } \\
\text { for } 12 \mathrm{~h} \text { after the procedure }\end{array}$ \\
\hline $\begin{array}{l}\text { Ozhan } \\
\text { et } a^{P^{5}}\end{array}$ & Atorvastatin & $\begin{array}{l}\text { High-dose atorvastatin } \\
80 \mathrm{mg} \text { plus } 600 \mathrm{mg} \mathrm{NAC} \\
\text { twice daily in } 1 \mathrm{st} \text { day } \\
\text { followed by } 80 \mathrm{mg} \\
\text { atorvastatin for } 2 \text { days after } \\
\text { procedure }\end{array}$ & $\begin{array}{l}\text { plus } 600 \mathrm{mg} \mathrm{NAC} \\
\text { twice daily in } 1 \mathrm{st} \text { day } \\
\text { followed by } 80 \mathrm{mg} \\
\text { atorvastatin for } 2 \text { days } \\
\text { after procedure }\end{array}$ & lopamidol & $97 \pm 7$ & $93 \pm 6$ & CAG & $\begin{array}{l}\text { IV } 0.9 \% \mathrm{NaCl} 1000 \mathrm{~mL} \\
\text { infusion during } 6 \mathrm{~h} \text { after } \\
\text { procedure }\end{array}$ \\
\hline Patti et a ${ }^{4}$ & Atorvastatin & $\begin{array}{l}80 \mathrm{mg} \text { atorvastatin } 12 \mathrm{~h} \\
\text { before intervention with } \\
\text { another } 40 \mathrm{mg} 2-\mathrm{h} \\
\text { preprocedure, after } \\
\text { procedure everyone put on } \\
40 \mathrm{mg} / \text { day }\end{array}$ & Placebo & lobitridol & $209 \pm 72$ & $213 \pm 13$ & $\mathrm{PCl}$ & $\begin{array}{l}\text { IV } 0.9 \% \mathrm{NaCl} \text { at } 1 \mathrm{~mL} / \mathrm{kg} / \mathrm{h} \\
\text { for } 12 \mathrm{~h} \text { before and } 12 \mathrm{~h} \\
\text { after intervention }\end{array}$ \\
\hline
\end{tabular}

Continued 


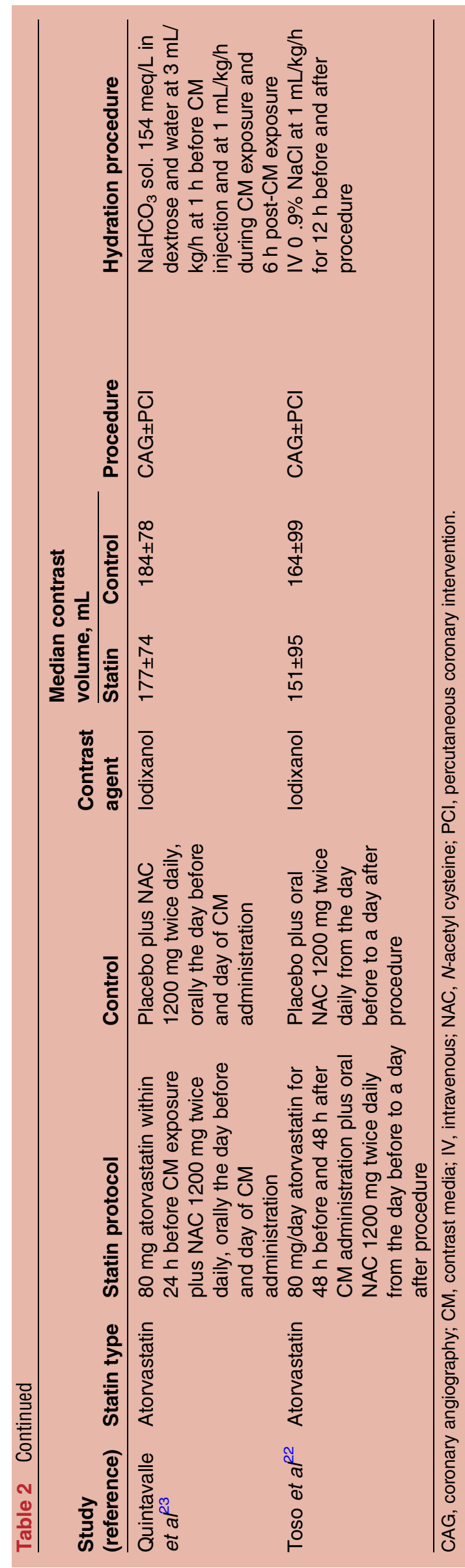

$<70 \mathrm{~min} / \mathrm{mL} / 1.73 \mathrm{~m}^{2}$. The definition of pre-existing renal impairment among the trials was not consistent. Four studies ${ }^{14} 222426$ included patients with serum creatinine clearance $<60 \mathrm{~mL} / \mathrm{min}$; and the remaining trials $^{13} 23$ only included GFR $>60 \mathrm{~mL} / \mathrm{min} / 1.73 \mathrm{~m}^{2}$. Therefore, our study defined renal impairment as GFR $<60 \mathrm{~mL} / \mathrm{min} / 1.73 \mathrm{~m}^{2}$ or creatinine clearance $<60 \mathrm{~mL} /$ min, excluding patients on dialysis.

In our subgroup analysis, we found that statins significantly reduced the risk of CIN in patientswith or without renal impairment (figure 4). In patients with renal impairment, statin reduced CIN risk by $54 \%$ $(\mathrm{MH}-\mathrm{RR}=0.46,95 \%$ CI 0.29 to $0.72, \mathrm{p}=0.0008)$. In patients without renal impairment, statin reduced the CIN risk by $55 \%$ (MH-RR $=0.42,95 \%$ CI 0.28 to 0.63 , $\mathrm{p}<0.0001)$. There was no statistically significant difference in the degree of beneficial effect of statin on CIN prevention between both groups $(\mathrm{p}=0.79)$.

Acute renal failure requiring dialysis in statin versus control group Out of nine RCTs, four trials ${ }^{13} 142226$ defined the number of patients who developed acute renal failure requiring dialysis. In Toso $e t a l^{22}$ and Jo $e t a l^{26}$ trials, the statin groups had no patients developing acute renal failure requiring dialysis, whereas the control groups had one event each. In two trials, ${ }^{14}{ }^{13}$ there were two events of acute renal failure requiring dialysis in the control group compared to none in the statin group. We analysed the relative risk of this major adverse event in the studies mentioned above, but it was not statistically significant (MH-RR=0.25, 95\% CI 0.05 to $1.21, \mathrm{p}=0.09$ ).

Quintavalle $e$ t $a l^{23}$ described the need for chronic dialysis in eight patients without differentiating its occurrence in either statin group or control group, from a total of 37 major events in 402 patients.

\section{Low-osmolar versus iso-osmolar contrast media}

The CIN prevention effect of statin therapy was also found to be independent of the type of contrast medium used (figure 5). Statin use significantly decreased the incidence of CIN by $48 \%$ in groups of patient who received isoosmolar contrast (MH-RR=0.52, 95\% CI 0.34 to 0.79 , $\mathrm{p}=0.002)$ and $64 \%$ in low-osmolar contrast $(\mathrm{MH}-\mathrm{RR}=0.31$, $95 \%$ CI 0.16 to $0.60, \mathrm{p}=0.0006)$. There was no statistically significant difference between the two groups in the degree of statin's CIN prevention effect $(\mathrm{p}=0.19)$.

\section{Concomitant pretreatment with NAC}

Among the nine RTC trials, four of the trials used NAC as a part of the study protocol in both treatment arm and control arm. Statin's CIN prevention effect was found to be independent of NAC use (figure 6). In patients without concurrent NAC therapy, statin reduced the risk of CIN by $49 \%$ (MH-RR $=0.51,95 \%$ CI 0.36 to $0.73, p=0.0002$ ). In patients who received NAC, statin reduced the risk of CIN by $54 \%(\mathrm{MH}-\mathrm{RR}=0.46,95 \%$ CI 0.25 to $0.83, \mathrm{p}=0.01)$. The efficacy of statins in prevention of CIN between both groups was not statistically different $(\mathrm{p}=0.74)$. 
Table 3 Clinical characteristics of the included studies

\begin{tabular}{|c|c|c|c|c|c|c|c|c|}
\hline \multirow[b]{2}{*}{ Study (reference) } & \multicolumn{2}{|c|}{ Mean age (years) } & \multicolumn{2}{|c|}{ Males (n) } & \multicolumn{2}{|c|}{$\begin{array}{l}\text { Diabetes } \\
\text { Mellitus (n) }\end{array}$} & \multicolumn{2}{|c|}{ Baseline creatinine (mg/dL)* } \\
\hline & Statin & Control & Statin & Control & Statin & Control & Statin & Control \\
\hline$\overline{\text { Acikel et } a f^{21}}$ & $58.7 \pm 8.5$ & $60.8 \pm 10.8$ & 51 & 51 & 19 & 20 & $0.84 \pm 0.14$ & $0.85 \pm 0.16$ \\
\hline Han et al ${ }^{13}$ & $61.45 \pm 8.64$ & $61.44 \pm 8.64$ & 963 & 991 & 1498 & 1500 & $1.1 \pm 0.25$ & $1.0 \pm 0.23$ \\
\hline Jo et $a^{26}$ & $65.0 \pm 9.3$ & $66.1 \pm 8.2$ & 91 & 88 & 35 & 29 & $1.29 \pm 0.42$ & $1.25 \pm 0.37$ \\
\hline Leoncini et $a l^{14}$ & $66.2 \pm 12.4$ & $66.1 \pm 13.5$ & 166 & 165 & 50 & 57 & $0.95 \pm 0.27$ & $0.96 \pm 0.28$ \\
\hline Li et $a{ }^{77}$ & $66.3 \pm 7.4$ & $65.4 \pm 7.2$ & 58 & 64 & 21 & 24 & $0.93 \pm 0.13$ & $0.93 \pm 0.13$ \\
\hline Ozhan et $a f^{55}$ & $54 \pm 10$ & $55 \pm 8$ & 27 & 40 & 9 & 12 & $0.88 \pm 0.20$ & $0.88 \pm 0.19$ \\
\hline Patti et a ${ }^{24}$ & $65 \pm 11$ & $66 \pm 10$ & 91 & 96 & 36 & 32 & $1.04 \pm 0.32$ & $1.04 \pm 0.22$ \\
\hline Quintavalle et $a^{R^{3}}$ & $70 \pm 6$ & $70 \pm 8$ & 103 & 120 & 89 & 80 & $1.32(0.96-1.62)$ & $1.29(0.88-1.61)$ \\
\hline Toso et $a^{22}$ & $75 \pm 8$ & $76 \pm 7$ & 104 & 92 & 31 & 33 & $1.20 \pm 0.35$ & $1.18 \pm 0.33$ \\
\hline
\end{tabular}

Table 4 Quality assessment of included randomised clinical trials

\begin{tabular}{|c|c|c|c|c|c|c|c|}
\hline Study & AC & $\mathbf{R}$ & Blinding & Analysis & FU completion & Similar base & I/E criteria \\
\hline Acikel et $a^{R^{1}}$ & UC & $Y$ & UC & UC & $\mathrm{Y}$ & $Y$ & $\mathrm{Y}$ \\
\hline Han et al ${ }^{13}$ & UC & $Y$ & UC & ITT & $Y$ & $Y$ & $Y$ \\
\hline Jo et $a^{26}$ & $\mathrm{Y}$ & $Y$ & DB & ITT & $Y$ & $\mathrm{Y}$ & $Y$ \\
\hline Leoncini et $a l^{14}$ & UC & $\mathrm{Y}$ & UC & UC & $\mathrm{Y}$ & $\mathrm{Y}$ & $Y$ \\
\hline Li et $a$ P7 $^{27}$ & $\mathrm{Y}$ & $Y$ & $\mathrm{BD}$ & UC & $\mathrm{Y}$ & $\mathrm{Y}$ & $Y$ \\
\hline Ozhan et $a{ }^{25}$ & UC & $\mathrm{Y}$ & UC & UC & $Y$ & $Y$ & $\mathrm{Y}$ \\
\hline Patti et $a R^{4}$ & $\mathrm{Y}$ & $\mathrm{Y}$ & DB & $\mathrm{PP}$ & $Y$ & $Y$ & $\mathrm{Y}$ \\
\hline Quintavalle et $a^{P^{3}}$ & $Y$ & $\mathrm{Y}$ & SB & ITT & $\mathrm{Y}$ & $Y$ & $Y$ \\
\hline Toso et $a^{P^{2}}$ & UC & $\mathrm{Y}$ & UC & UC & $\mathrm{Y}$ & $Y$ & $Y$ \\
\hline
\end{tabular}

Statin type (comparison between atorvastatin and rosuvastatin)

From the nine RCT, there were six studies that used atorvastatin and two newer trials Han $e t a l^{13}$ and Leoncini $e t a l^{14}$ used rosuvastatin. The beneficial effect of statin was seen in patients who received atorvastatin (MH-RR $=0.38,95 \%$ CI 0.21 to $0.71, \mathrm{p}=0.002$ ) as well as patients who received rosuvastatin (MH-RR $=0.53,95 \%$ CI 0.38 to $0.74, \mathrm{p}=0.0002$; figure 7).
Diabetes status

In our subgroup analysis, we compared the outcome of interest (CIN) based on diabetes mellitus status (figure 8). The risk reduction of CIN in patients with diabetes was $39 \%$ (MH-RR $=0.61,95 \%$ CI 0.43 to $0.86, \mathrm{p}=0.005)$, and in patients without diabetes was $39 \%(\mathrm{MH}=\mathrm{RR}=0.61,95 \% \mathrm{CI}$ 0.33 to $1.13, \mathrm{p}=0.11$ ). However, the difference in risk reduction of CIN was not statistically significant between both groups $(\mathrm{p}=0.99)$.

\begin{tabular}{|c|c|c|c|c|c|c|c|c|}
\hline Study or Subgroup & Statin & in & $\begin{array}{l}\text { Contr } \\
\text { Events }\end{array}$ & $\begin{array}{l}\text { ol } \\
\text { Total }\end{array}$ & Weight & $\begin{array}{c}\text { Risk Ratio } \\
\text { M-H, Random, } 95 \% \mathrm{Cl}\end{array}$ & \multicolumn{2}{|c|}{$\begin{array}{c}\text { Risk Ratio } \\
\text { M-H, Random, } 95 \% \mathrm{Cl}\end{array}$} \\
\hline Acikel $S$ et al., 2010 & 0 & 80 & 1 & 80 & $1.1 \%$ & $0.33[0.01,8.06]$ & & \\
\hline Han Y et al., 2014 & 34 & 1498 & 58 & 1500 & $25.4 \%$ & $0.59[0.39,0.89]$ & 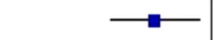 & \\
\hline Jo SH et al., 2008 & 3 & 118 & 4 & 118 & $4.5 \%$ & $0.75[0.17,3.28]$ & & \\
\hline Leoncini $M$ et al., 2014 & 17 & 252 & 38 & 252 & $19.8 \%$ & $0.45[0.26,0.77]$ & & \\
\hline Li W et al., 2012 & 2 & 78 & 13 & 83 & $4.6 \%$ & $0.16[0.04,0.70]$ & & \\
\hline Ozhan $\mathrm{H}$ et al., 2010 & 2 & 60 & 7 & 70 & $4.2 \%$ & $0.33[0.07,1.54]$ & & \\
\hline Patti $G$ et al., 2011 & 6 & 120 & 16 & 121 & $10.2 \%$ & $0.38[0.15,0.93]$ & & \\
\hline Quintavalle C et al.,2012 & 9 & 202 & 37 & 208 & $14.6 \%$ & $0.25[0.12,0.51]$ & & \\
\hline Toso A et al., 2010 & 15 & 151 & 16 & 152 & $15.6 \%$ & $0.94[0.48,1.84]$ & & \\
\hline Total $(95 \% \mathrm{Cl})$ & & 2559 & & 2584 & $100.0 \%$ & $0.47[0.34,0.65]$ & & \\
\hline Total events & 88 & & 190 & & & & & \\
\hline $\begin{array}{l}\text { Heterogeneity: } \operatorname{Tau}^{2}=0.0 \\
\text { Test for overall effect: } Z=\end{array}$ & $\begin{array}{l}\mathrm{Chi}^{2}=1 \\
49(P \leq 0\end{array}$ & $\begin{array}{l}1.25, \mathrm{df} \\
.00001\end{array}$ & $=8(P=$ & 19); & $=299$ & & $\begin{array}{cc}1 & 1 \\
0.2 & 0.5 \\
\text { Favors Statin }\end{array}$ & $\begin{array}{rrr}1 & 1 & 1 \\
& 2 & 5 \\
\text { Favors Control }\end{array}$ \\
\hline
\end{tabular}

Figure 2 Forest plot of risk ratios with its $95 \% \mathrm{Cl}$ for the incidence of $\mathrm{CIN}$ among patients taking statin versus control. 


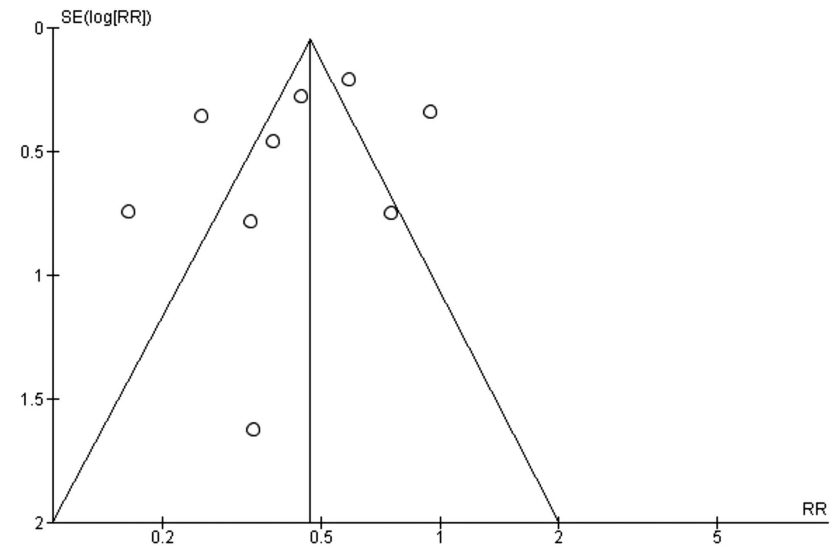

Figure 3 Funnel plot with its $95 \% \mathrm{Cl}$ to evaluate the evidence of publication bias.

\section{DISCUSSION}

This meta-analysis based on nine randomised controlled trials demonstrates that administration of statin therapy has a statistically significant benefit of preventing CIN among patients undergoing interventional procedures requiring contrast media when compared to placebo or NAC. In addition, both rosuvastatin and atorvastatin has shown statistically beneficial role in preventing CIN.

CIN is a recognised complication after contrast media administration during interventional procedures. The beneficial effect of statins on CIN prevention may be explained by multiple mechanisms. After exposure to contrast medium, there is a short period of vasodilation followed by intense renal vasoconstriction. Various molecules, including angiotensin, appear to be involved in the mechanism of this renal hypoperfusion. ${ }^{31}$ Statins down-regulate angiotensin receptors and decrease endothelin synthesis, and therefore may prevent renal hypoperfusion and ischaemia. ${ }^{32}$ Contrast medium may also cause direct damage to tubular cells facilitated by oxygen-free radicals, proinflammatory cytokines and complement activation, leading to protein precipitates, which causes tubular obstruction. ${ }^{31}$ Statins have antioxidant and anti-inflammatory properties as well as tendency to reduce endothelin secretion, which may be the mechanism for the CIN prevention effect of statins. ${ }^{33} 34$

Our present study adds to the current literature by suggesting that statins prevent CIN even in patients with pre-existing renal impairment. Previous studies and meta-analysis were not able to find any CIN preventive effect of statins in patients with chronic kidney disease. ${ }^{9} 22 \quad 26 \quad 35$ This is most likely because of the smaller sample size of patients being studied and analysed. We included a total of 1330 patients with renal impairment in our subgroup analysis of the CIN prevention effect of statins. This is concordant with other studies showing the beneficial effects of statins in patients with CKD. ${ }^{36}{ }^{37}$ However, given that most of our included trials have excluded patients with GFR $<30$, it remains unclear if statins will be beneficial in reducing CI-AKI in patients with CKD stage 4 or 5 . We also found that there were more patients who developed CIN, who eventually required dialysis in the control group as compared to the group who received statin therapy (six out of 2022 vs none out of 2019), although conclusions from this observation cannot be made due to incomplete reporting of ultimate progression to haemodialysis from several of the studies.

Our analysis also showed that the CIN preventive effect of statins is seen in patients receiving iso-osmolar

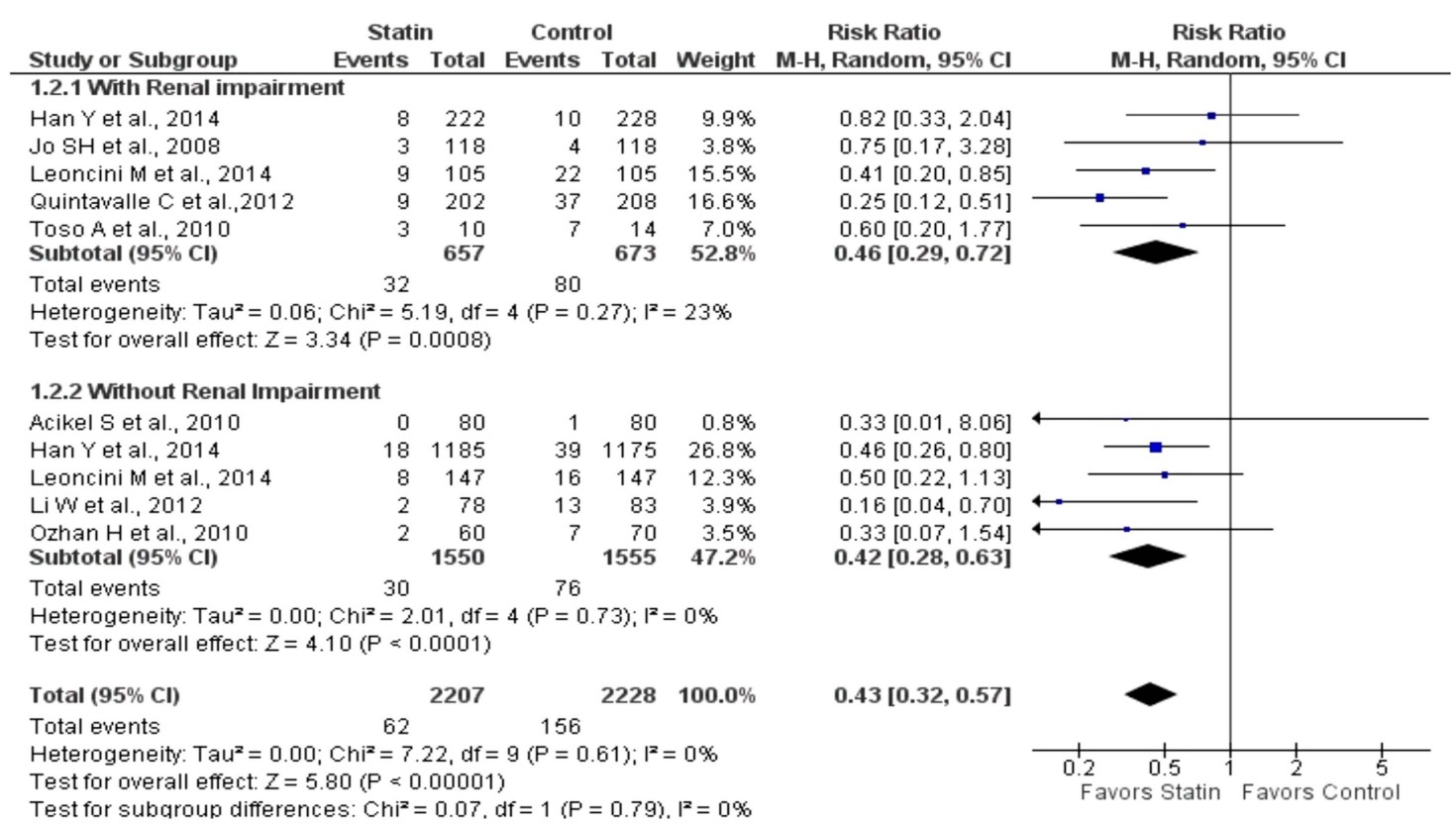

Figure 4 Forest plot of risk ratio with its $95 \% \mathrm{Cl}$ for the incidence of contrast-induced nephropathy among patient taking statin versus control based on renal impairment status (with renal Impairment vs without renal impairment). 


\begin{tabular}{|c|c|c|c|c|c|c|c|c|}
\hline$\frac{\text { Study or Subgroup }}{\text { 1.3.1 lodixanol Use }}$ & $\begin{array}{l}\text { Stati } \\
\text { Events }\end{array}$ & Total & $\begin{array}{l}\text { Contr } \\
\text { Events }\end{array}$ & ol & Weight & $\begin{array}{l}\text { Risk Ratio } \\
\text { M-H, Random, } 95 \% \mathrm{Cl}\end{array}$ & \multicolumn{2}{|c|}{$\begin{array}{c}\text { Risk Ratio } \\
\text { M-H, Random, } 95 \% \mathrm{Cl}\end{array}$} \\
\hline Han $Y$ et al., 2014 & 34 & 1498 & 58 & 1500 & $25.4 \%$ & $0.59[0.39,0.89]$ & & \\
\hline Leoncini $M$ et al., 2014 & 17 & 252 & 38 & 252 & $19.8 \%$ & $0.45[0.26,0.77]$ & & \\
\hline Quintavalle C et al.,2012 & 9 & 202 & 37 & 208 & $14.6 \%$ & $0.25[0.12,0.51]$ & & \\
\hline Toso A et al., 2010 & 15 & 151 & 16 & 152 & $15.6 \%$ & $0.94[0.48,1.84]$ & & \\
\hline \multicolumn{9}{|c|}{$\begin{array}{l}\text { Heterogeneity: } \operatorname{Tau}^{2}=0.11 ; \mathrm{Chi}^{2}=8.10, \mathrm{df}=4(\mathrm{P}=0.09) ; \mathrm{I}^{2}=51 \% \\
\text { Test for overall effect: } Z=3.06(\mathrm{P}=0.002)\end{array}$} \\
\hline \multicolumn{9}{|l|}{ 1.3.2 No lodixanol Use } \\
\hline Acikel $S$ et al., 2010 & 0 & 80 & 1 & 80 & $1.1 \%$ & $0.33[0.01,8.06]$ & & \\
\hline Li W et al., 2012 & 2 & 78 & 13 & 83 & $4.6 \%$ & $0.16[0.04,0.70]$ & & \\
\hline \multicolumn{9}{|c|}{$\begin{array}{l}\text { Heterogeneity: } \text { Tau }^{2}=0.00 ; \mathrm{Chi}^{2}=0.95, \mathrm{df}=3(\mathrm{P}=0.81) ; \mathrm{I}^{2}=0 \% \\
\text { Test for overall effect: } Z=3.45(\mathrm{P}=0.0006)\end{array}$} \\
\hline Total $(95 \% \mathrm{Cl})$ & & 2559 & & 2584 & $100.0 \%$ & $0.47[0.34,0.65]$ & & \\
\hline Total events & 88 & & 190 & & & & & \\
\hline $\begin{array}{l}\text { Heterogeneity: } \operatorname{Tau}^{2}=0.0 \\
\text { Test for overall effect: } Z= \\
\text { Test for subqroup differer }\end{array}$ & $\begin{array}{l}\text { Chi }{ }^{2}=11 \\
49(P=0 \\
\text { es: } \text { Chi }^{2}=\end{array}$ & $\begin{array}{l}1.25, \mathrm{df} \\
.00001) \\
=1.73 . \mathrm{d}\end{array}$ & $\begin{array}{l}=8(P=1 \\
d f=1(P=\end{array}$ & $0.19) ; \mathrm{I}^{2}$ & $\begin{array}{l}=29 \% \\
I^{2}=42.3\end{array}$ & & $\begin{array}{cc}0.2 & 0.5 \\
\text { Favors Statin }\end{array}$ & $\begin{array}{ccc}1 & 1 \\
& 2 & 5 \\
\text { Favors Control }\end{array}$ \\
\hline
\end{tabular}

Figure 5 Forest plot of risk ratio with its $95 \% \mathrm{Cl}$ for the incidence of contrast-induced nephropathy among patient taking statin versus control based on contrast medium use (iodixanol vs non-iodixanol).

or low-osmolar contrast. This finding is in keeping with the 2012 Kidney Disease: Improving Global Outcomes (KDIGO) guidelines, ${ }^{2}$ which recommend both options of contrast (low-osmolal or iso-osmolar) without a precedence of one over the other. ${ }^{38}$ Looking at the absolute reduction of CIN in our analysis, low-osmolar contrast had a lower incidence of CIN. However, this absolute reduction of CIN might be attributed to the reduced average amount of contrast used when compared to isoosmolar contrast.
Risk factors for CIN include CKD, use of first generation hyperosmolal ionic contrast agents, hypovolemia, non-steroidal anti-inflammatory drug (NSAID) use, renal transplantation and heart failure. ${ }^{39-41}$

In our subgroup analysis, we found the CIN prevention effect of statins was independent of the use of NAC. It has been previously suggested that statins would not benefit patients who had been premedicated with NAC, with the hypothesis that both statins and NAC act on similar pathways. ${ }^{9}$ Our findings suggest that patients may

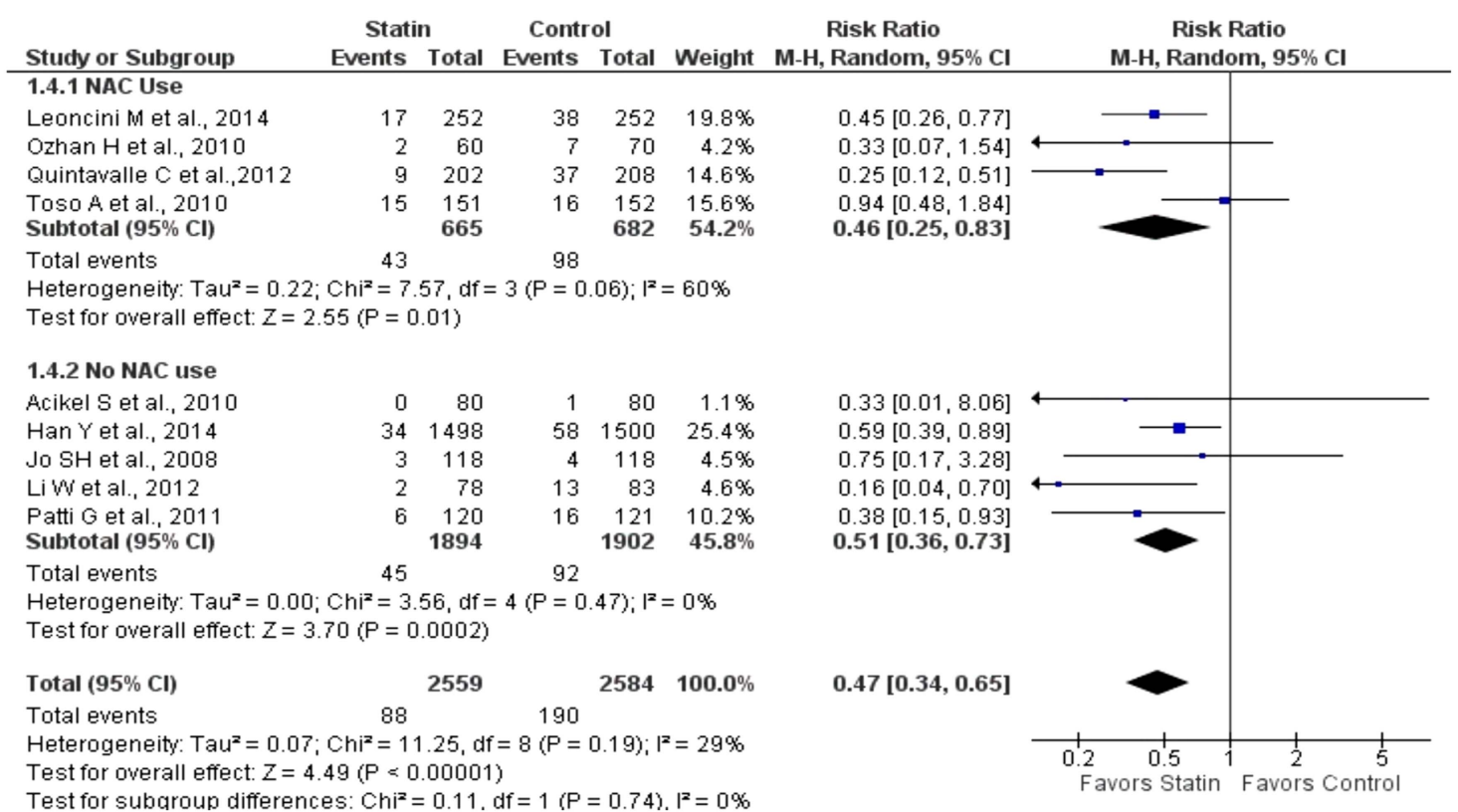

Figure 6 Forest plot of risk ratio with its $95 \% \mathrm{Cl}$ for the incidence of contrast-induced nephropathy among patient taking statin versus control based on $N$-acetyl cysteine (NAC) use (NAC use vs no NAC use). 


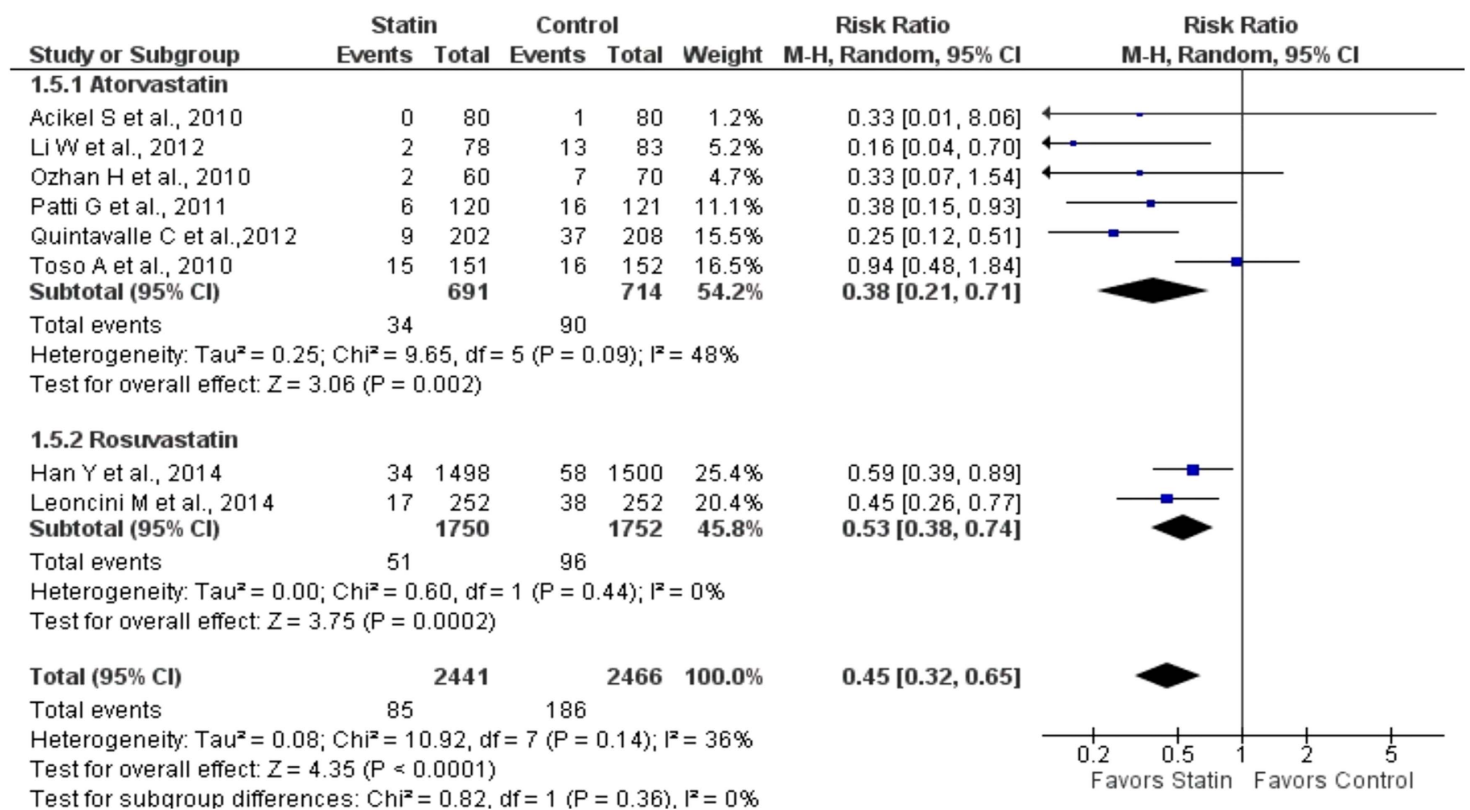

Figure 7 Forest plot of risk ratio with its $95 \% \mathrm{Cl}$ for the incidence of contrast-induced nephropathy among patient taking statin versus control based on statin type (atorvastatin vs rosuvastatin).

benefit from administration of statins regardless of whether they were planned to receive NAC or not.

We also found that the beneficial effects of statins were seen equally in patients that were treated with high-dose atorvastatin and rosuvastatin. Definitive conclusions regarding the superiority of one of the two mentioned statins over the other cannot be made. The question of whether simvastatin exerts the same CIN prevention effect remains unclear, especially when the RCTs using simvastatins are limited. In Jo et $a l,{ }^{26}$ simvastatin did not show a statistical significant decrease risk of CIN. This raises the question on the role of statin potency in the mechanism of CIN prevention.

Our study yielded a significant result in statin's CIN prevention efficacy in patients with diabetes. Our nonsignificant result in patients with no diabetes is most likely due to inadequate sample size in the group with no diabetes, as our subgroup analysis included 3228 patients with diabetes and only 914 patients without diabetes. Supporting this theory is that both groups were found to have similar RRs of 0.61 .

There are several limitations in this meta-analysis. First, during the analysis, we found that most of the

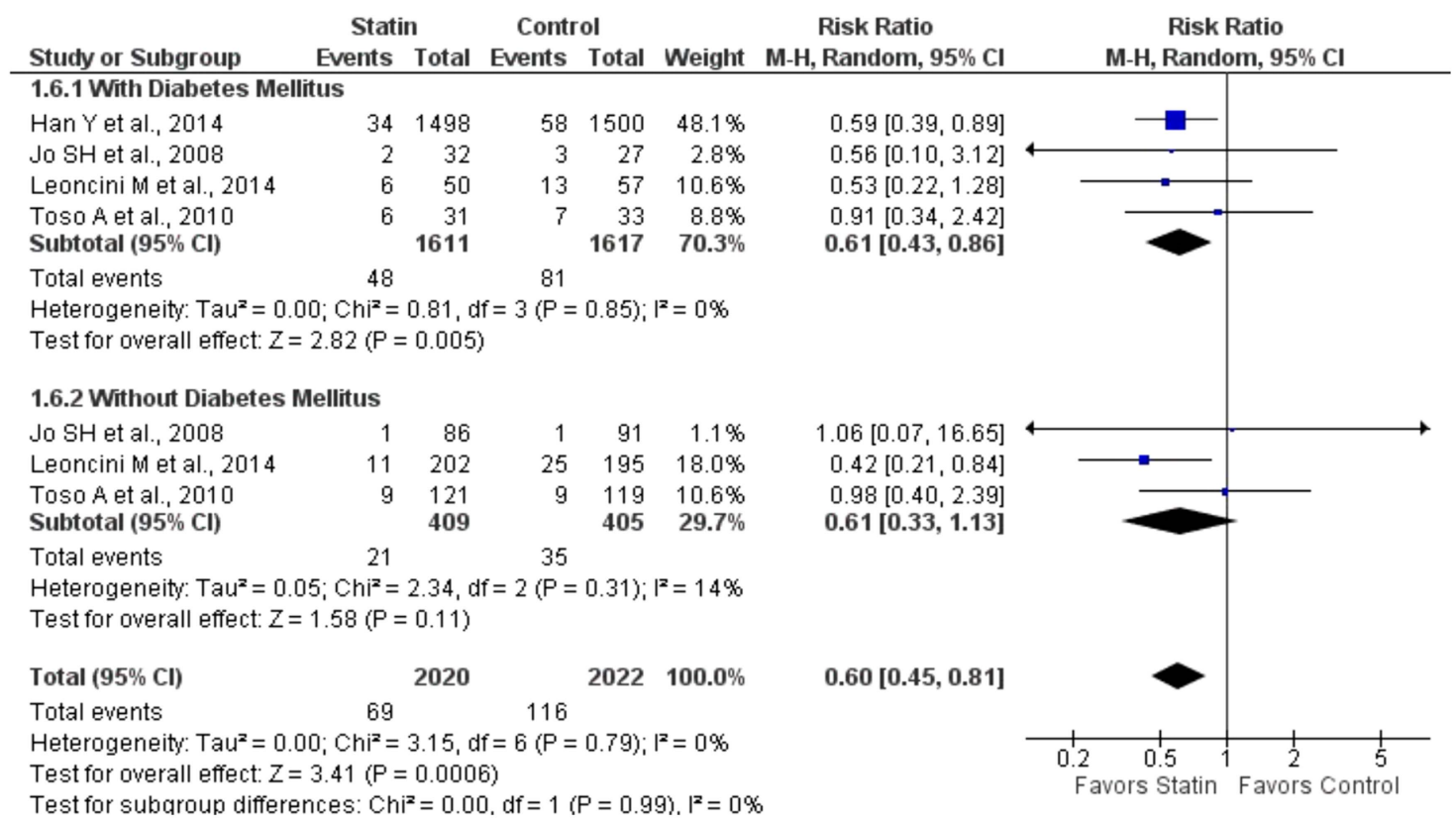

Figure 8 Forest plot of risk ratio with its $95 \% \mathrm{Cl}$ for the incidence of contrast-induced nephropathy among patient taking statin versus control based on diabetes mellitus status (with diabetes mellitus vs without diabetes mellitus). 
randomised controlled trials of statin use as prevention of CIN were performed in patients undergoing coronary angiography or PCI. We are unaware of randomised trials examining statin use for the prevention of CIN outside of cardiovascular angiography. Given likely preexisting use in this patient cohort, the finding of statin benefit is unlikely to have large clinical implication. There clearly exists a need to repeat these trials in patients who are likely to be statin naive, undergoing iodinated contrast exposure for non-cardiovascular imaging procedures such as CT scan.

Second, the studies analysed the CIN prevention effect of statins with a total of three types of statins, at varied doses, for different duration of treatment. The latest two studies used rosuvastatin for $12 \mathrm{~h}$ prior to the procedure, and were continued for 12 and $24 \mathrm{~h}$ in each study, respectively. These variations in treatment protocols may have lead to different effects in prevention of CIN. Third, the definition of CIN was not the same in all the studies and varied in the amount of absolute increase of creatinine and under different predetermined post-procedural time period.

\section{CONCLUSION}

The results of this meta-analysis suggest that statin pretreatment reduces the incidence of CIN, and is independent presence of renal impairment, of the type of contrast used and concomitant NAC therapy. Further studies are needed to determine the appropriate statin pretreatment regimen, including the type, dosing and duration of statin pretreatment. Our study sets a platform for future pharmacological guidelines and clinical trials, as well as highlights the need for trials studying statin's efficacy in other non-cardiac contrast procedures such as radiological imaging.

\section{Author affiliations}

${ }^{1}$ Department of Cardiovascular Diseases, University of Arizona, Tucson, Arizona, USA

${ }^{2}$ Department of Internal Medicine, University of Arizona, Tucson, Arizona, USA

${ }^{3}$ Arizona Health Sciences Library, University of Arizona, Tucson, Arizona, USA

${ }^{4}$ Department of Cardiovascular Disease, Mayo Clinic, Phoenix, Arizona, USA

Contributors NS, JZL and JJH were involved in integration of data and drafting of manuscript. SWL took part in revision of the manuscript and integration of data. $\mathrm{CH}$ was involved in systematic search of the literature. AP took part in integration of data and revision of manuscript. PS took part in revising the manuscript critically for important intellectual content. KSL was involved in conception and design, revising critically for important intellectual content and final approval of the manuscript.

Funding This research received no specific grant from any funding agency in the public, commercial or not-for-profit sectors.

Competing interests None.

Provenance and peer review Not commissioned; externally peer reviewed.

Data sharing statement No additional data are available.

Open Access This is an Open Access article distributed in accordance with the Creative Commons Attribution Non Commercial (CC BY-NC 4.0) license, which permits others to distribute, remix, adapt, build upon this work noncommercially, and license their derivative works on different terms, provided the original work is properly cited and the use is non-commercial. See: http:// creativecommons.org/licenses/by-nc/4.0/

\section{REFERENCES}

1. Nash K, Hafeez A, Hou S. Hospital-acquired renal insufficiency. Am J Kidney Dis 2002;39:930-6.

2. Khwaja A. KDIGO clinical practice guidelines for acute kidney injury. Nephron Clin Pract 2012;120:c179-84.

3. Solomon R, Dauerman HL. Contrast-induced acute kidney injury. Circulation 2010;122:2451-5.

4. Brown JR, Malenka DJ, DeVries JT, et al. Transient and persistent renal dysfunction are predictors of survival after percutaneous coronary intervention: insights from the Dartmouth Dynamic Registry. Catheter Cardiovasc Interv 2008;72:347-54.

5. Goldenberg I, Chonchol M, Guetta V. Reversible acute kidney injury following contrast exposure and the risk of long-term mortality. Am J Nephrol 2009;29:136-44.

6. McCullough PA, Wolyn R, Rocher LL, et al. Acute renal failure after coronary intervention: incidence, risk factors, and relationship to mortality. Am J Med 1997;103:368-75.

7. Levine GN, Bates ER, Blankenship JC, et al. 2011 ACCF/AHA/SCAI guideline for percutaneous coronary intervention a report of the American College of Cardiology Foundation/American Heart Association Task Force on Practice Guidelines and the Society for Cardiovascular Angiography and Interventions. Circulation 2011;124: e574-651.

8. Tumlin J, Stacul F, Adam A, et al. Pathophysiology of contrast-induced nephropathy. Am J Cardiol 2006;98:14K-20.

9. Li Y, Liu Y, Fu L, et al. Efficacy of short-term high-dose statin in preventing contrast-induced nephropathy: a meta-analysis of seven randomized controlled trials. PLoS ONE 2012;7:e34450.

10. Zhang BC, Li WM, Xu YW. High-dose statin pretreatment for the prevention of contrast-induced nephropathy: a meta-analysis. Can J Cardiol 2011;27:851-8.

11. Zhang L, Zhang L, Lu Y, et al. Efficacy of statin pretreatment for the prevention of contrast-induced nephropathy: a meta-analysis of randomised controlled trials. Int J Clin Pract 2011;65:624-30.

12. Pappy R, Stavrakis S, Hennebry TA, et al. Effect of statin therapy on contrast-induced nephropathy after coronary angiography: a meta-analysis. Int J Cardiol 2011;151:348-53.

13. Han Y, Zhu G, Han L, et al. Short-term rosuvastatin therapy for prevention of contrast-induced acute kidney injury in patients with diabetes and chronic kidney disease. J Am Coll Cardiol 2014;63:62-70.

14. Leoncini M, Toso A, Maioli M, et al. Early high-dose rosuvastatin for contrast-induced nephropathy prevention in acute coronary syndrome: results from the PRATO-ACS study (protective effect of rosuvastatin and antiplatelet therapy on contrast-induced acute kidney injury and myocardial damage in patients with acute coronary syndrome). J Am Coll Cardiol 2014;63:71-9.

15. Briguori CMDP, Visconti GMD, Rivera NVP, et al. Cystatin C and contrast-induced acute kidney injury. Circulation 2010;121:2117-22.

16. Verhagen AP, de Vet HC, de Bie RA, et al. The Delphi list: a criteria list for quality assessment of randomized clinical trials for conducting systematic reviews developed by Delphi consensus. J Clin Epidemiol 1998;51:1235-41.

17. Liberati A, Altman DG, Tetzlaff J, et al. The PRISMA statement for reporting systematic reviews and meta-analyses of studies that evaluate health care interventions: explanation and elaboration. PLoS Med 2009;6:e1000100.

18. Higgins JPT, Thompson SG, Deeks JJ, et al. Measuring inconsistency in meta-analyses. BMJ 2003;327:557-60.

19. DerSimonian R, Laird N. Meta-analysis in clinical trials. Control Clin Trials 1986;7:177-88.

20. Begg CB, Mazumdar M. Operating characteristics of a rank correlation test for publication bias. Biometrics 1994;50:1088-101.

21. Acikel S, Muderrisoglu H, Yildirir A, et al. Prevention of contrast-induced impairment of renal function by short-term or long-term statin therapy in patients undergoing elective coronary angiography. Blood Coagul Fibrinolysis 2010;21:750-7.

22. Toso A, Maioli M, Leoncini M, et al. Usefulness of atorvastatin (80 $\mathrm{mg}$ ) in prevention of contrast-induced nephropathy in patients with chronic renal disease. Am J Cardiol 2010;105:288-92.

23. Quintavalle C, Fiore D, De Micco F, et al. Impact of a high loading dose of atorvastatin on contrast-induced acute kidney injury. Circulation 2012;126:3008-16. 
24. Patti G, Ricottini E, Nusca A, et al. Short-term, high-dose Atorvastatin pretreatment to prevent contrast-induced nephropathy in patients with acute coronary syndromes undergoing percutaneous coronary intervention (from the ARMYDA-CIN [atorvastatin for reduction of myocardial damage during angioplasty contrast-induced nephropathy] trial. Am J Cardiol 2011;108:1-7.

25. Ozhan H, Erden I, Ordu S, et al. Efficacy of short-term high-dose atorvastatin for prevention of contrast-induced nephropathy in patients undergoing coronary angiography. Angiology 2010;61:711-14.

26. Jo SH, Koo BK, Park JS, et al. Prevention of radiocontrast medium-induced nephropathy using short-term high-dose simvastatin in patients with renal insufficiency undergoing coronary angiography (PROMISS) trial-a randomized controlled study. Am Heart J 2008;155:499.e1-8.

27. Li W, Fu X, Wang Y, et al. Beneficial effects of high-dose atorvastatin pretreatment on renal function in patients with acute ST-segment elevation myocardial infarction undergoing emergency percutaneous coronary intervention. Cardiology 2012;122:195-202.

28. Kellum JA, Lameire N, Aspelin P, et al. Work group membership. Kidney Int 2012;2:1.

29. Higgins JPT, Thompson SG. Quantifying heterogeneity in a meta-analysis. Stat Med 2002;21:1539-58.

30. Higgins JP, Thompson SG, Deeks JJ, et al. Measuring inconsistency in meta-analyses. BMJ 2003;327:557-60.

31. Goldenberg I, Matetzky S. Nephropathy induced by contrast media: pathogenesis, risk factors and preventive strategies. CMAJ 2005; 172:1461-71.

32. Bonetti PO, Lerman LO, Napoli C, et al. Statin effects beyond lipid lowering —are they clinically relevant? Eur Heart J 2003;24:225-48.
33. Wierzbicki AS, Poston R, Ferro A. The lipid and non-lipid effects of statins. Pharmacol Ther 2003;99:95-112.

34. Zager RA, Johnson AC, Hanson SY. Radiographic contrast media-induced tubular injury: evaluation of oxidant stress and plasma membrane integrity. Kidney Int 2003;64:128-39.

35. Zhang L, Gong D, Li S, et al. Meta-analysis of the effects of statin therapy on endothelial function in patients with diabetes mellitus. Atherosclerosis 2012;223:78-85.

36. Ridker PM, MacFadyen J, Cressman M, et al. Efficacy of rosuvastatin among men and women with moderate chronic kidney disease and elevated high-sensitivity C-reactive protein: a secondary analysis from the JUPITER (Justification for the Use of Statins in Prevention-an Intervention Trial Evaluating Rosuvastatin) trial. J Am Coll Cardiol 2010;55:1266-73.

37. Abe M, Maruyama N, Yoshida Y, et al. Efficacy analysis of the lipid-lowering and renoprotective effects of rosuvastatin in patients with chronic kidney disease. Endocr J 2011;58:663-74.

38. Lameire N, Kellum JA, for the KAKIGWG. Contrast-induced acute kidney injury and renal support for acute kidney injury: a KDIGO summary (Part 2). Crit Care 2013;17:205.

39. Cronin R. Contrast-induced nephropathy:pathogenesis and prevention. Pediatr Nephrol 2010;25:191-204.

40. Parfrey PS, Griffiths SM, Barrett BJ, et al. Contrast material-induced renal failure in patients with diabetes mellitus, renal insufficiency, or both. A prospective controlled study. N Engl J Med 1989;320:143-9.

41. Mehran R, Aymong ED, Nikolsky E, et al. A simple risk score for prediction of contrast-induced nephropathy after percutaneous coronary intervention: development and initial validation. J Am Coll Cardiol 2004;44:1393-9. 